\title{
Theoretical Analysis of the Influence of Asperity's Dimensions Affected by a Scale Factor on the Mixed Lubrication between Parallel Surfaces
}

\author{
F. Robbe-Valloire $\mathbb{i}^{1},{ }^{1}$ R. Progri, ${ }^{1,2}$ and T. Da Silva Botelho ${ }^{1}$ \\ ${ }^{1}$ QUARTZ (EA 7393), Supmeca, 3 rue Fernand Hainaut, 93407 Saint-Ouen, France \\ ${ }^{2}$ Retired Supmeca Professor, France \\ Correspondence should be addressed to F. Robbe-Valloire; francois.robbe-valloire@supmeca.fr
}

Received 18 January 2018; Revised 19 April 2018; Accepted 3 May 2018; Published 19 June 2018

Academic Editor: Michael M. Khonsari

Copyright (C) 2018 F. Robbe-Valloire et al. This is an open access article distributed under the Creative Commons Attribution License, which permits unrestricted use, distribution, and reproduction in any medium, provided the original work is properly cited.

\begin{abstract}
Mixed lubrication between a given pair of surfaces is directly related to the parameter $\eta u / p$. Any change in microgeometry produces a shift in the transition between the boundary and lubricated regimes. Using an asperity based model including five families of asperities ( 2 for full fluid lubrication and 3 for local boundary lubrication), we simulate mixed lubricated behavior. Our theoretical results confirm the relation between the mixed regime and the $\eta u / p$ parameter. All homothetic changes in microgeometry affecting the vertical and horizontal directions by the same scaling factor Sc induce a shift in the transition. The most interesting result is that this shift is exactly the same if speed $u$ is scaled by $1 / \mathrm{Sc}$ with the initial microgeometry. This particular behavior, which is in good agreement with experimental results proposed in the literature, comes from the fact that behavior at each asperity can be written using dimensionless parameters. Most of these parameters are independent to any scaling of the microgeometry and only one, the speed parameter, needs to be artificially scaled in order to remain unchanged by the modification of the microgeometry.
\end{abstract}

\section{Introduction}

Mixed lubrication is a specific regime of lubrication characterized by an increasing friction coefficient (and wear) compared to that classically obtained when the fluid film properly separates both surfaces in contact (full lubrication regime). This increasing friction coefficient is related to the existence of part of contact where local film thickness can be so thin (on the order of few molecules of oil) that speed accommodation through the film cannot occur. As a consequence of this behavior, one can observe increasing friction (and wear). This situation takes place at the summit of the highest asperities, and, because of the classical range of microgeometry amplitude, it may be possible to observe a local continuous film on the other part of the contact pair, particularly at the lowest asperities. This physical aspect clearly shows the link between mixed lubrication and microgeometry, but despite the large amount of literature devoted to this relationship, it is not fully understood, in particular for large flat contacts. The main cause for this gap in understanding appears to be the number of diverse geometric configurations of lubricated contacts and the specificities of calculation associated with each one of them.

Local point and concentrated line contacts are probably the most studied typologies of lubricated rough contact. Theoretical studies about such contact are generally based on the well-known deterministic method using a mesh to model the microgeometry. The limited area of contact permits a deterministic description of the microgeometry and makes this numerical method well suited for studies of fluid perturbation in the case of a noncontact situation and/or contact under more severe conditions. A bibliography synthesis proposed by Hooke [1] in 2005 gives illustration of the number of publications using this numerical method. More recently, some works try to associate numerical Reynold's based analysis for fluid part of the interface with analytical statistical analysis of direct contact [2]. Concentrated contacts, such as that used in rolling contact between ball bearings or gears, 
generally working in rolling / sliding cinematic condition, are not considered in the present work. In this study, we only consider large flat contact.

Concerning large conformal contact, there is a lack of research on general relationship between mixed lubrication and microgeometry. Many studies are primarily devoted to evaluating the modification of the hydrodynamic effect induced by microgeometry. Only a few studies have also undertaken analysis of direct contact and the corresponding friction enhancement. The classical deterministic method cannot be directly used to model this situation because of the large number of points along the dimension of contact and the resolution needed for a microgeometry description. A number of studies have addressed the specific case of journal bearing using the deterministic method and have proposed evolutions of the method. Dobrica [3] suggests numerical simplification in order to reduce the number of numerical operations. Finite Element Method analysis, for both fluid and solid analysis, using a commercial finite element analysis software, is proposed by Albers [4], but only on a small representative part of the bearing. A coupled analysis using statistical analysis for direct contact and average flow Reynolds formulation is proposed by Zhang [5] for studying tribological effect of texturing in mixed lubrication. Multiscale analysis of defects was proposed by Fricke [6]. In this study, influence of surface form defect is calculated on the whole bearing, while microgeometry effect is quantifying using a representative small area of the bearing.

For mixed lubrication induced between parallel flat surfaces, publications including experimental results are very rare, particularly concerning the influence of microgeometry. Key articles on the subject are proposed by Emmens [7] and Felder [8], who present experimental based studies of the influence of microgeometry in the context of parallel surfaces. Both authors perform tribological tests of flat contact in the deep drawing of steel sheets where several microgeometries are present. Both authors confirm a mixed lubrication transition directly related to the microgeometry of the contacting surfaces. For the same set of contact parameters, the friction coefficient is reduced by a decrease in the height of microgeometry. Quantitatively, they suggest new parameter associations that include surface roughness based on the classical parameter $\eta u / p$. On the basis of their experiments, the use of these parameters gives a better localization of the points near a single curve (or master curve) than the use of the classical parameter $\eta u / p$. Emmens [7] proposes $H_{e}=\eta u / p R_{p}{ }^{2}$ and adds the $R_{p}$ parameter (altitude of the mean line of the profile from the lowest point). This new parameter $H_{e}$ is homogeneous of a length. Emmens suggests that this length could be related to the periodicity of the microgeometry defects. Felder [8] proposed the dimensionless parameter $H_{f}=3 \eta l u / p R_{a}{ }^{2}$, where $R_{a}$ is the arithmetic mean value and $l$ is the space between two consecutive asperities. He proposes this expression in order to obtain a parameter in the form of $p_{l} / p$, where $p_{l}$ is the theoretical pressure induced by the pouches generated by the microgeometry. This theoretic pressure is obtained assuming a perfect regular microgeometry and Rayleigh's pad shape. More recently, Nogueira [9] completed these experimental studies, comparing the mixed lubrication behavior of various materials (steels, copper alloys) that have different microgeometries. In addition to the microgeometry effect, she observes a significant influence exerted by the mechanical properties of materials in contact. The best correlation of the experimental results is obtained using Emmens' parameter with a term that includes the elastic properties of the contacting materials $H_{n}=H_{e} / E q^{2 / 3}$, where $\mathrm{Eq}$ is the equivalent Young's modulus of the contacting materials.

Existing theoretical approaches proposed for mixed lubrication in parallel flat surfaces mostly concern the case of externally pressurized contact used in the field of dynamic faced sealing. Lebeck [10] offers a wide-ranging survey of possible mechanisms and analyses effects such as undesirable pad tilting or thermal deformation or the squeeze effect coupling with dynamic behavior of the supports. But the most promising approach appears to be the asperity lubrication effect known as the microhydrodynamic effect. More recently, authors [11] attempt to integrate the statistical description of microgeometry, and the contact behavior is studied using the Greenwood-Williamson theory. All contacting asperities undergo plastic deformation while behavior for the noncontacting area is obtained using the classic Reynold's equation, including Patir and Cheng's flow factor, in order to take into account the effect of roughness on fluid flow.

Theoretical approach for parallel flat surfaces in contact without external pressurization is mainly devoted to the field of sheet metal forming. Methodology coupled numerical average flow Reynolds based analysis for fluid and statistical analysis for deformed asperities. In this case deformation regime of asperities is also only plastic [12]. Specific evolution takes into account flattening of workpiece asperities and ploughing induced by tool asperities [13].

For this situation of parallel lubricated contact without oil pressurization, we seek to construct the behavior of the contact also using the statistical analysis but by association of the local behavior of each asperity [14], in order to take into account contact situations where elastic to plastic deformation occurs. Contacting asperities can be assigned to one of three regimes (elastic, elastoplastic, or full plastic regimes) depending on the level of indentation [15]. Noncontacting asperities work with hydrodynamic lubrication or elastohydrodynamic lubrication depending on the level of elastic deformation. In the following, we aim to determine whether theoretical analysis can confirm the influence of microgeometry on mixed lubrication between parallel surfaces. This paper deals only with effects induced by a scaling of the microgeometry. This kind of modification affects by similar amplification both the vertical and lateral directions of the microgeometry. In our methodology, we study a specific case and simulate the scaling of microgeometries tested in the same condition (Section 3). The surprisingly consistent shift of the mixed lubrication transition, obtained with the performed scaling of the microgeometry, suggests a theoretical explication based on a geometrical dimensionless formulation detailed in Section 2. 


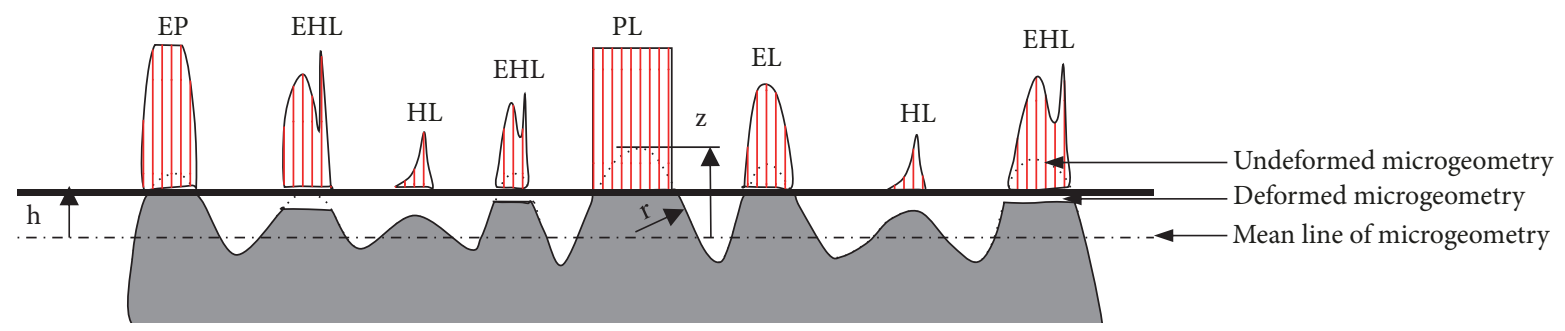

FIGURE 1: The five lubrication regimes at the asperity scale.

\section{Description of the Model}

The mixed lubrication model proposed in past articles by the authors has not undergone any modification [14]. Accordingly, in this section we only provide a brief outline of the model, discussing its behavior in order to highlight the effect of a scaled microgeometry on mixed lubrication.

The basis of this mixed lubrication model is to extend Greenwood's theory initially proposed for dry contact between nominally flat rough surfaces, to lubricated rough contact. This is made by association of the individual normal load calculated at each asperity for a given separation (or mean oil thickness) $h$ between the surfaces. A test and trial method, based on the h separation variable, can identify the separation able to generate a given normal load at the point of contact. Concerning the normal load at each asperity, we have detected for a given contact the possibility of 5 families of asperities (Figure 1) depending on the local geometry of the asperity. The specific mechanism for each of the 5 families depends on the value of the local indentation of the asperity ( $\mathrm{z}$-h value). For each family, a normal analytical expression is proposed. The aim of each paragraph in this section is to present this expression and to discuss the possibility for its use in a geometrically dimensionless formula.

The different families are the following:

(i) Elastic deformation (EL): this situation concerns asperities without a local fluid effect subjected to a normal load level, keeping all the materials in the linear reversible elastic deformation mechanism.

(ii) Elastoplastic deformation $(E P)$ : owing to the indentation effect, local stress can exceed the yield stress of the material. The contact mechanism is called elastoplastic deformation because both elastic and plastic deformation take place. The degree of plastic deformation increases with the degree of indentation.

(iii) Full plastic deformation $(P L)$ : this mechanism occurs when plastic deformation is generalized across the whole area of contact.

(iv) Isoviscosity and piezoviscosity hydrodynamic lubrication $(H L)$ : pressure in the fluid film is low and insufficient to cause elastic deformation of the asperity. For large film thickness, the calculation is based on the assumption of rigid bodies with isoviscous fluid. On decreasing the film thickness, the fluid pressure increases and the initial effect is a change of viscosity, which is described by the classical exponential Barus' law of piezoviscosity.

The association of elementary normal load is a simple summation and, as a consequence, neglected interaction between neighboring asperities is a necessary assumption of the calculation. Of course due to the very large number of asperities present in the contact, simple summation can be advantageously replaced by probability analysis using a statistical description of the microgeometry. The variables used for the statistical description of microgeometry must be the same as that used in the expression of individual normal load: $z$, the altitude of asperity. If $F_{\text {regime }}(z)$ is the expression of the normal load on an asperity working with the considered regime (EL, EP, PL, HL, or EHL regimes), we suggest to adopt the well-known load sharing concept, firstly proposed by Johnson [16], where total load is sharing between contacting asperities and lubricating film. We only extend it to the five families of asperity we have identified. As a consequence, the total normal load $\mathrm{N}$ acting on all these asperities is expressed as

$$
\begin{aligned}
N & =n_{0} \int_{z_{\min }}^{z_{\max }} f(z) * F_{\text {regime }}(z) d z \\
& =N_{E L}+N_{E P}+N_{P L}+N_{H L}+N_{E H L}=N_{b}+N_{l}
\end{aligned}
$$

In this relation, $n_{0}$ is the total number of asperities in the considered area of contact $\mathrm{A}_{0}\left(n_{0}=\beta \mathrm{A}_{0}\right.$ with $\beta$ density of asperities), and $f(z)$ is the probability to have an asperity at the altitude z. $N_{\text {regime }}$ (subscript regime can be $E L, E P, P L$, $H L$, and $E H L)$ are normal loads supported by asperities in the considered regime. $N_{b}=N_{E L}+N_{E P}+N_{P L}$ is normal load supported by all asperities in the local boundary regime and $N_{f}=N_{H L}+N_{E H L}$ is the normal load for asperities in the local fluid regime.

Friction force in the considered contact is induced by both contributions: $T=T+T_{f}$, but it is important to note that $T_{\mathrm{f}}$ can be generally neglected due to the very low friction at full fluid sites compared to that produced at boundary lubricated sites in a mixed lubrication regime. Assuming a mean friction coefficient $f_{b}$ for boundary lubrication, friction force at the contact can be expressed as

$$
T \cong f_{b} N_{b}
$$




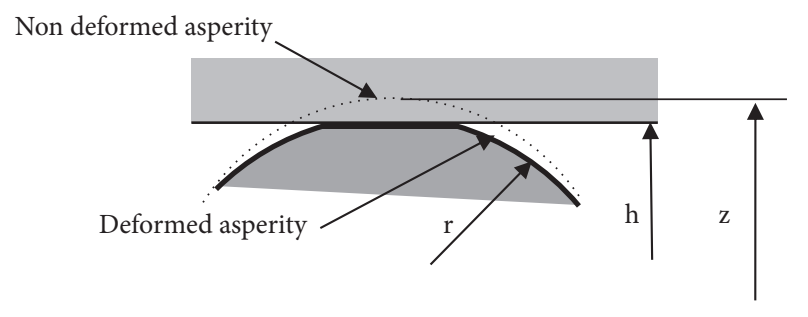

FIGURE 2: Local boundary contact.

Consequently, an approximation of the friction coefficient in mixed lubrication is

$$
f \cong f_{b} \frac{N_{b}}{N}=f_{b} \phi
$$

$\phi=N_{b} / N=\left(N_{E L}+N_{E P}+N_{P L}\right) / N$ is the ratio of the normal load transmitted by the local boundary lubrication. This ratio appears as a governing factor for friction coefficient evaluation in a mixed lubrication regime. Numerical values of this ratio, obtained in a given situation of scaled microgeometries, will be studied in the case detailed in Section 3 . First, however, the analytical expression of individual normal load at asperities will be presented using a geometrically dimensionless expression.

2.1. Elastic Deformation Regime. The local boundary situation refers to asperities in direct contact with the antagonist. A necessary condition is that the altitude of the undeformed asperity $z$ is greater than the altitude $h$ of the antagonist (Figure 2). In this condition, if we neglect the boundary film thickness, we obtain $\delta=z-h$ as the indentation value.

The elastic deformation regime is a first local boundary situation and corresponds to the lowest values for the indentation $\delta$ and, in this case, the behavior is based on the Hertz theory for a ball-on-plan contact. The relationship between the normal load and the indentation is expressed as

$$
F_{E L}=\frac{2 E_{q}}{3} r^{1 / 2} \delta^{3 / 2}
$$

$E_{q}=2 /\left[\left(1-v_{1}^{2}\right) / E_{1}+\left(1-v_{2}^{2}\right) / E_{2}\right]$ corresponds to the equivalent Young modulus of both materials in contact and $r$ is the radius of asperity. In order to facilitate a general discussion of mixed lubrication, we suggest the use of dimensionless parameters such as $\Delta=\delta / r$. We obtain

$$
F_{E L}=\frac{2 E_{q} r^{2}}{3} \Delta^{3 / 2}
$$

Using a dimensionless parameter for the normal load, $W_{E L}=F_{E L} / E_{q} r^{2}$, yields

$$
W_{E L}=\frac{2}{3} \Delta^{3 / 2}
$$

As suggested by authors [17, 18], the limit of the elastic deformation regime corresponds to the appearance of plasticity in the contact, and assuming a Tresca criterion, we obtain for the critical indentation $\delta_{\text {Elim }}$

$$
\frac{\delta_{E L \lim }}{r}=\Delta_{E L \lim }=\left(\frac{0.5 \pi}{0.3} \frac{R p_{e}}{E q}\right)^{2} \approx 27.4 \frac{R p_{e}^{2}}{E q^{2}}
$$

We can observe that, in elastic regime, the normal load and limit of this regime can be easily expressed using geometrical dimensionless parameter. If the indentation is greater than this limit (asperity could have a greater altitude or a smaller radius), elasticity and plasticity coexist in the contact. Equation (4) ceases to be applicable and the regime becomes elastoplastic.

2.2. Elastoplastic Deformation Regime (EP). In this case, elastic and plastic deformations coexist in the contact. The ratio between the two kinds of deformation depends on the contact conditions. We use theoretical results first proposed by Hill and reported by Johnson [17]. These results refer to plastic deformation caused by a uniform pressure imposed by a spherical indenter. The corresponding equation for the normal load is

$$
F_{E P}=\frac{2 \pi}{3} R p_{e} a^{2}\left[1.80+\ln \left(\frac{1}{6} \frac{E_{q}}{R p_{e}} \frac{a}{r}\right)\right]
$$

Chang et al. [18] propose a link between the radius $a$ of the contact area and the indentation $\delta$. This link is based on the classical assumption of matter conservation during plastic deformation. Redistribution of matter is localized near the contact and preserves a rounded shape:

$$
a^{2}=r\left[2 \delta-\delta_{\text {ELlim }}\right]
$$

This elastoplastic behavior can also be formulated using the dimensionless parameters $W_{E P}$ (resp., $\Delta$ ) for normal load (resp., indentation):

$$
\begin{aligned}
W_{E P}= & \frac{2 \pi}{3} \frac{R p_{e}}{E_{q}}\left(2 \Delta-\Delta_{\text {ELlim }}\right) \\
& \cdot\left[1.80+\ln \left(\frac{E_{q}}{R p_{e}} \frac{\sqrt{2 \Delta-\Delta_{\text {ELlim }}}}{6}\right)\right]
\end{aligned}
$$

This mechanism continues to be applicable until plasticity occupies all the contact area to produce the so-called plasticity regime.

The use of a finite element model $[19,20]$ for this situation shows that the assumption of a constant pressure is not really adapted. Uniform pressure underestimates finite element results by up to $38 \%$ for intermediate interference. Another interesting result of these finite element studies [19, $20]$ is that solution can be expressed with empirical curve fitting including 2 stages of power laws expressions using dimensionless parameters deduced from both parameters we use in relation (10): $\Delta$ and $\Delta_{\text {ELlim }}$. Such results indicate that a way of improvement of quantitative results can be performed with new law but conclusion of this work, directly based on the existence of laws based on dimensionless parameters, as explained in Section 3.4, could not change by using new set of dimensionless relation. 


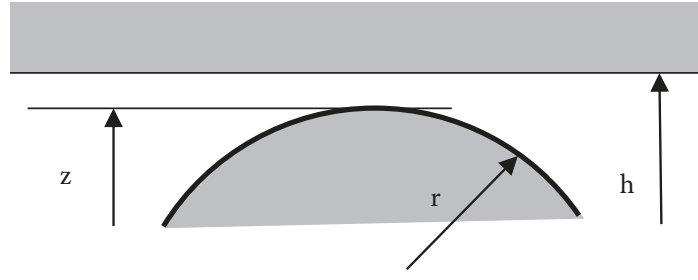

FIgURE 3: Local boundary contact.

2.3. Full Plastic Deformation Regime (PL). The plastic deformation mechanism corresponds to a generalized plastic deformation in the contact. Thus the mean contact pressure $p_{m}$ remains constant at $p_{m}=3 R p_{e}$. As regards elastoplastic deformation, we use a matter conservation assumption based on Chang's equation relating indentation and the radius of the contact area. The results can be also written using the dimensionless parameters:

$$
W_{P L}=3 \pi \frac{R p_{e}}{E q}\left(2 \Delta-\Delta_{E L l i m}\right)
$$

Assuming continuity with the elastoplastic domain, full plastic deformation begins at the interference:

$$
\Delta_{\text {EPlim }}=\frac{\delta_{E P l i m}}{r} \cong 3980\left(\frac{R p_{e}}{E_{q}}\right)^{2}
$$

2.4. Fluid Lubricated Hydrodynamic Regime (HL). Fluid lubricated asperity concerns asperity where a continuous film separates both surfaces. $h_{0}=(h-z)$ is the film thickness of this contact (Figure 3 ) and by coherence with the notations used in Sections 2.1-2.3, we suggest the use of the geometrical dimensionless parameter $H_{0}=(h-z) / r$.

This first domain corresponds to a fluid contact situation and is characterized by the highest local values of the fluid film thickness. In order to obtain a local fluid film inside the contact, a necessary condition is that the summit of the considered asperity is below the altitude of the antagonist. This first domain also corresponds to the furthest asperities from the counterpart. The assumptions made are the following:

(i) The solids are rigid.

(ii) The viscosity of the fluid follows Barus' law: $\eta=$ $\eta_{0} \exp [\alpha * p]$, where $\alpha$ is the piezoviscosity coefficient and $p$ the local pressure in the lubricant fluid.

As for the previous regimes, an analytical solution can be proposed using a two-step calculation. Firstly, calculation is made using assumption of a constant viscosity for the lubricant (IVR isoviscous regime). The solution [21] is expressed as

$$
W_{I V R}=8.1 \frac{U}{H_{0}^{0.5}}
$$

In this relation, $\mathrm{U}$ is the dimensionless speed parameter: $U=\eta u / 2 E_{q} r$ and $W_{I V R}=F_{I V R} / E_{q} r^{2}$.
The application of Barus' law in Reynold's equation assumes a simple relationship between local pressures in the piezoviscous (HL) and the isoviscous regimes (IVR). As a consequence, the dimensionless normal load, using piezoviscous regime, can be approached [14] by

$$
W_{H L}=W_{I V R}\left[1-0.194 \ln \left(1-\frac{1.05 G U}{H_{o}^{3 / 2}}\right)\right]
$$

In this relation, $\mathrm{G}$ is the dimensionless material parameter $G=\alpha E_{q}$. Fortunately, in this new regime, relation uses the dimensionless parameters. Quantitative analysis of the evolution of the normal load shows a rapid increase at a given value $H_{o H L l i m}$. In this case, the assumption of rigid solids becomes unacceptable and the elastohydrodynamic regime begins. Accordingly, we choose to stop using the piezoviscous hydrodynamic regime near this value. The validity domain for the hydrodynamic situation is

$$
H_{o}=\frac{h-z}{r} \geq H_{o H L l i m}=1.126(G U)^{2 / 3}
$$

2.5. Fluid Lubricated Elastohydrodynamic Regime (EHL). If the dimensionless film thickness is lower than the previously proposed value, the large increase in pressure induced by the rigid asperity suggests that the regime becomes elastohydrodynamic. Due to the spherical shape of asperity, the formula proposed by Hamrock and Dowson [22] seems applicable; however, the problem with a normal load evaluation is to identify the value of the film thickness because it should be influenced by elastic deformation of the asperity. If we neglect the existence of the final convergent at the end of the EHL contact, and if we suppose a constant film thickness corresponding to the central film thickness deduced from the Hamrock and Dowson equation for circular contact, we obtain the following equations:

$$
\begin{aligned}
& H_{c}=1.916 U^{0.670} G^{0.53} W_{E H L}^{-0.067} \\
& H_{c}=\Delta+H_{o}
\end{aligned}
$$

As proposed by Grubin, we suggest the determination of the indentation $\Delta$ using the Hertz equation deduced from dry elastic contact $\left(W_{E H L} \cong W_{E L}\right)$. The corresponding assumption is that the film thickness is low compared to the indentation. $\Delta$ and $H_{c}$ can be eliminated in (17), using (6) and (16). We obtain

$$
1.916 U^{0.67} G^{0.53} W_{E H L}^{-0.067}=\left(\frac{3 W_{E H L}}{2}\right)^{2 / 3}+H_{o}
$$

This relation immediately yields the expression of $H_{0}$ (position of the asperity) as a function of $W_{E H L}$ (normal load at the considered asperity). But unfortunately, this equation cannot be inversed in an analytical way. A second-order approximation for the solution of this equation gives

$$
\begin{aligned}
& W_{E H L} \\
& \quad=\frac{2}{3}\left[H_{P}\left(1-0.922 \frac{H_{0}}{H_{P}}+0.007365\left(\frac{H_{0}}{H_{P}}\right)^{2}\right)\right]^{3 / 2}
\end{aligned}
$$

with $H_{p}=1.81\left(G^{0.48} U^{0.61}\right)$ and $H_{o}=(h-z) / r$. 


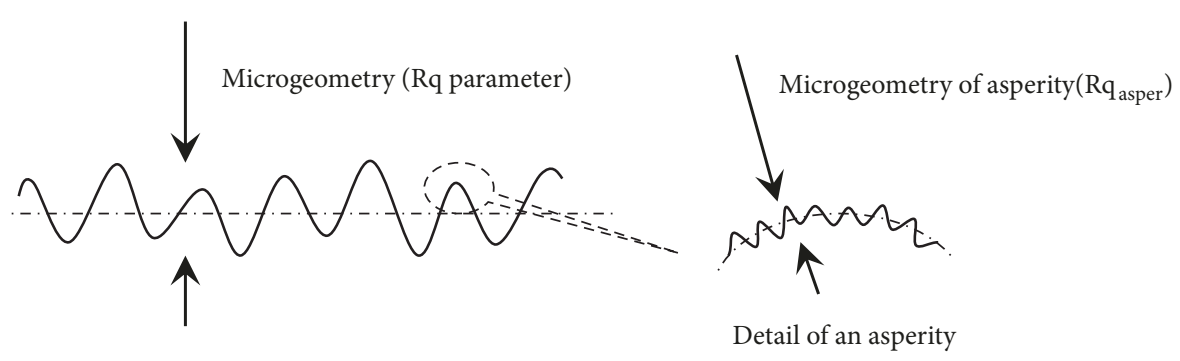

FIGURE 4: Definition of the microgeometry of asperities.

The limit of this elastohydrodynamic regime corresponds to a film thickness too thin compared to the height of roughness [14]. If we name $R q_{\text {asper }}$ the roughness of the asperities (Figure 4), consequently, this criterion is expressed as $h_{\text {cEHLlim }}=1.5 R q_{\text {asper }}$.

If $h_{c} \geq 1.5 R q_{\text {asper }}$, the mean film thickness can be sufficient to maintain a continuous fluid film. We obtain

$$
H_{\text {oEHLlim }}=-\left(\frac{1.97 U^{0.67} G^{0.53}}{R q_{\text {asper }} / r}\right)^{9.95}
$$

\section{Theoretical Results and Discussion}

In this section, we seek to illustrate the effect of microgeometry using an example. We compare several lubricated contacts with the same characteristics (listed in Table 1) except for their microgeometry.

Note that all parameters represent a fixed value except the speed parameter $u$ (in the range of 0.1 to $2 \mathrm{~m} / \mathrm{s}$ ) which is used as variable for characterizing the mixed lubrication domain.

The studied microgeometry is presented in Figure 5. This isotropic microgeometry is measured on a $2.5 \mathrm{~mm} \mathrm{x} 2.5$ $\mathrm{mm}$ area of a specimen manufactured by shoot preening. Roughness parameter for this surface is $\mathrm{Rq} \sim 2.8 \mu \mathrm{m}$.

In the following, we consider scaled microgeometries deduced from the reference microgeometry (presented in Figure 5) by a homothetic factor $S_{c}$ in the vertical direction and also in the horizontal direction (Figure 6). As a consequence, the vertical roughness parameters are scaled by the same factor Sc. The same scaling is observed for horizontal parameters, including mean periodicity between asperities, and, finally, for parameters such as the radius of asperities.

3.1. Statistical Description of the Microgeometry. As proposed by Greenwood and Williamson [23], we adopt a normal distribution for the peak heights $z$ and a constant radius $r$ for all asperities. Peak distribution uses the mean value $m_{\mathrm{z}}$ and the standard deviation $s_{\mathrm{z}}$ to express the peak altitude.

$$
f_{1}(z)=\frac{1}{s_{z} \sqrt{2 \pi}} \exp \left[-\frac{1}{2}\left(\frac{z-m_{z}}{s_{z}}\right)^{2}\right]
$$

A key issue is the determination of both parameters for this normal distribution. The method for determining $m_{z}, s_{z}$, and $r$ is explained in another article [24]. By applying this method, we can obtain the parameters presented in Table 2.
TABLE 1: Parameters of the studied contact.

\begin{tabular}{lc}
\hline Parameters & Nominal \\
\hline Contact & Smooth on rough $\left(30 \mathrm{~mm}^{2}\right.$ area $)$ \\
Lubricant & Newtonian fluid \\
Speed & $\begin{array}{l}\eta=100 \text { Pas and } \alpha=2.010-8 \mathrm{~Pa}^{-1} \\
\text { Microgeometry }\end{array}$ \\
Mean apparent pressure & Rough on $\mathrm{Smooth}$ \\
Materials & $1 \mathrm{MPa}$ \\
Steel & Steel on Brass \\
Brass & $E_{1}=210^{11} ; v_{1}=0.3$ \\
& $E_{2}=110^{11} ; v_{2}=0.3$ \\
Boundary friction coefficient & $R p_{e}=500 \mathrm{MPa}$ \\
& $f_{b}=0.12$
\end{tabular}

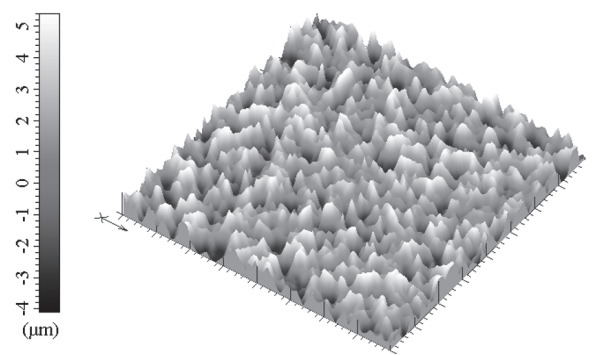

FIGURE 5: The reference microgeometry.

Finally, as proposed in the theoretical part, the microgeometry description must be completed by a description of the surface relief of the asperities. To achieve a quantitative evaluation of this surface relief for each asperity, we use a filtering technique adjusted to suppress the shape of the asperity. As presented in [14], the crucial point is the determination of the high pass cut-off. We propose to quantify this microrelief using the root mean square (RMS) standardized parameter $R q$. This value $\left(R q_{\text {asperity }}=1.4 \mu \mathrm{m}\right.$ for the proposed microgeometry) will be used in the next section as data for the description of the transition from local boundary lubrication to local continuous fluid lubrication.

3.2. Stribeck Curve. Figure 7 presents 3 Stribeck curves (friction coefficient versus $\eta u / p$ parameter) calculated using 
TABle 2: Parameters of the statistical description.

\begin{tabular}{lcc}
\hline Parameters & Definition & Value \\
\hline$m_{z}$ & Mean altitude for summit from reference line & $4.25 \mu \mathrm{m}$ \\
$s_{z}$ & Standard deviation for asperity summit & $1.80 \mu \mathrm{m}$ \\
$r$ & Radius of asperities & $400 \mu \mathrm{m}$ \\
$\beta$ & Density of asperities & $18 \mathrm{~mm}^{-2}$ \\
\hline
\end{tabular}

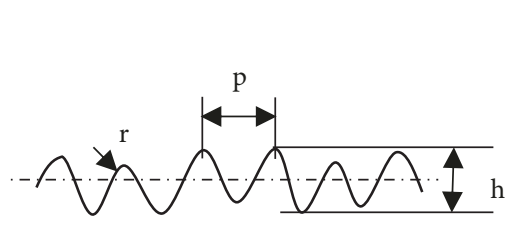

(a) Reference microgeometry

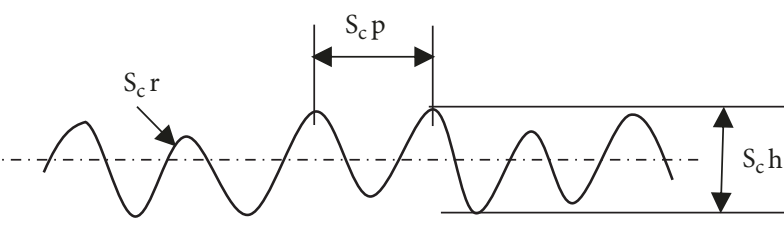

(b) Example of a scaled microgeometry

FIGURE 6: Principle of a scaled microgeometry.

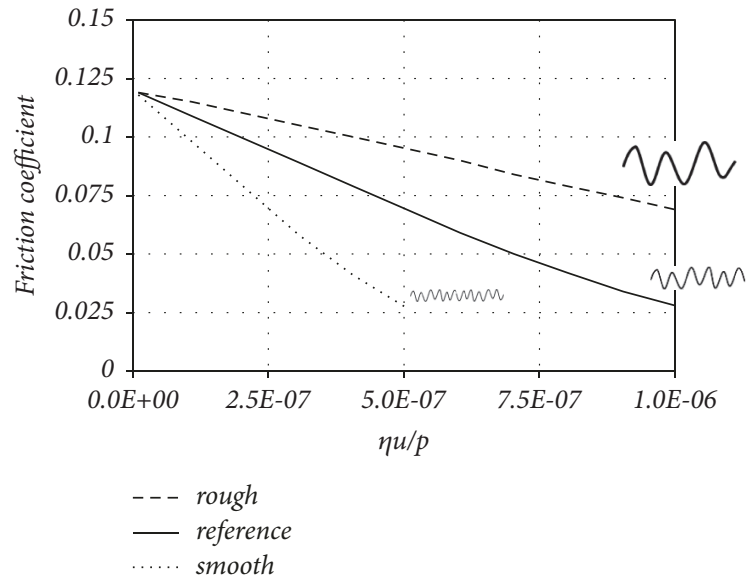

FIGURE 7: Stribeck curves obtained for the reference condition and influence of the microgeometry.

the methodology proposed in Section 2 and obtained with the same condition of contact (detailed in Table 1), but with three different microgeometries. The reference microgeometry corresponds to the microgeometry presented in Figure 5. Other curves, labeled rough or smooth, correspond to homothetic microgeometries. The rough and smooth microgeometries are deduced from the reference using a magnification or scale factor of 2 (for the rough geometry) and $1 / 2$ (for the smooth geometry). Consequently, the rough microgeometry shows asperities with a double height and a double periodicity compared to the reference's asperities, whereas the asperities on the smooth surface are half the height and half the thickness of the asperities in the reference microgeometry.

According to the assumption used in (2), calculations are only performed for the domain where boundary lubrication remains significant compared to full fluid lubrication (friction coefficient ranging from 0,125 to 0,025 ).

As can be observed, the model correctly predicts the shape of the evolution in the friction coefficient with the $\eta u / p$ parameter and suggests that a large influence is exerted

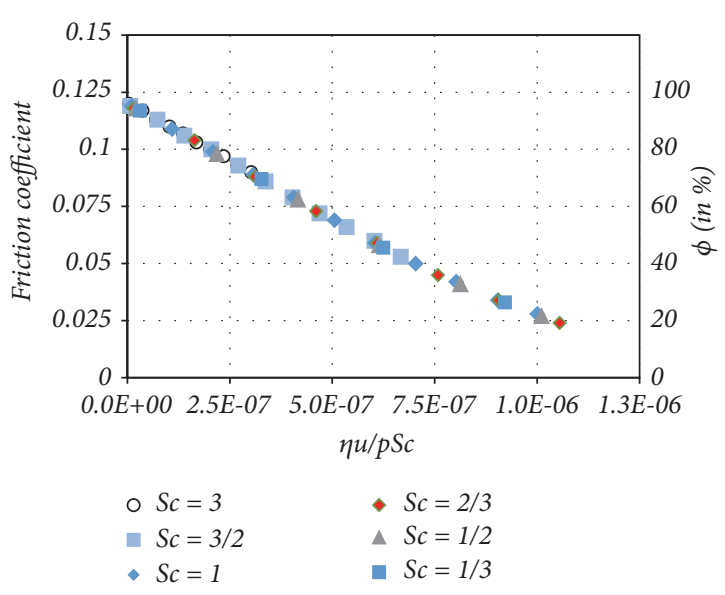

FIGURE 8: Stribeck curve obtained in the reference condition showing the influence of the microgeometry.

by the microgeometry of the surface. As expected, in a mixed lubrication regime, an increasing $\eta u / p$ parameter decreases the friction coefficient. Decreasing the amplitude of the microgeometry also has this effect. More precisely, with respect to the microgeometry, we notice that the scale factor $S_{\mathrm{c}}$ plays a significant role because a factor of 2 (or $1 / 2$ ) is sufficient to completely modify the Stribeck curve. In addition, a smooth microgeometry reduces the domain of mixed lubrication.

In order to compare the impact of the microgeometry scale factor $S_{c}$ compared to that of the Stribeck parameter $\eta u / p$, we plot the calculated evolution of the friction coefficient in mixed lubrication using a new parameter $\eta u / p S_{c}$, which tries to include the microgeometry effect with the scale factor $S_{c}$ (Figure 8).

From a quantitative perspective, it is interesting to note that the use of the scale factor in the denominator of the Stribeck parameter induces perfect superposition of all points on a single curve and consequently suggests an envelope curve for the shape of the considered microgeometry. Two 
remarks result from this perfect organization over a wide range of scale parameters (1/3 to 3$)$ and the Stribeck parameter:

(i) A comparison with experimental results proposed in the literature

(ii) A theoretical analysis of this perfect single curve obtained with microgeometries with asperities modified by a scale factor

3.3. Confrontation with Existing Experimental Results. This envelope curve for the theoretical results can be compared to the experimental results obtained by Emmens [7] and Felder [8]. Both authors perform experimental study using various microgeometries and propose the inclusion of the microgeometry effect in a modified Stribeck parameter, with slight differences from one author to the other: Emmens proposes $H_{e}=3 \eta u / p R_{p}{ }^{2}$, where $R_{p}$ is the altitude of the mean line of the profile from the lowest point. However, in the final discussion, the author explains that something is missing in this equation because this parameter has a dimension of length. Emmens suggests that the missing entity could be a parameter describing the periodicity of the asperity. As a consequence, the microgeometry parameter proposed by Emmens is proportional to $l / R p^{2}$. Application of a scale factor $S c$ to the microgeometry affects the numerator by a factor $S c$ (coming from l) and the denominator by $S c^{2}$ (coming from $R p^{2}$ ). Equivalent parameter for microgeometry deduced from such experimental works is $1 / S c$ in the case of scaled microgeometries, so the same we have obtained in our theoretical developments.

Meanwhile, Felder proposes $H_{f}=3 \eta l u / p R_{a}{ }^{2}$, where $R_{a}$ is the arithmetic mean and $l$ describes the space between two consecutive asperities. The proposed parameter is dimensionless, and if we applied the scale effect to this parameter, we can observe that $H_{f}$ is affected by $S_{c}$ at the numerator (due to the $l$ parameter) and $S_{\mathrm{c}}{ }^{2}$ at the denominator due to the term $R a^{2}$. It is important to note that the global effect $\left(1 / S_{c}\right)$ also exactly corresponds to the effect shown by the model we have developed.

3.4. Theoretical Aspects. The second remark is an explanation for the perfect shape of the envelope curve obtained with homothetic microgeometries. In fact, as proposed in Section 2, all theoretical expressions (normal load, variables, limits, etc.) are formulated using dimensionless parameters. The examination of these parameters shows that most of them are insensitive to the scale effect in the microgeometry:

(1) $Z=(z-m) / s_{z}$ is the dimensionless variable describing the altitude of summits; $m_{\mathrm{z}}$ and $s_{\mathrm{z}}$ are proportional to the scale factor of the microgeometry and, consequently, if the variable $\mathrm{z}$ is chosen proportional to the scale factor, the dimensionless parameter $Z$ is independent of $S_{c}$.

(2) Both the indentation parameter $\Delta=(z-h) / r$ and the local film thickness parameter $H_{0}=(h-z) / r$ include $z$ and $r$ variables that are proportional to the scale factor $S_{\mathrm{c}}$. If we impose a $h$ value proportional to the scale factor, both of these dimensionless parameters are independent of the microgeometry scale factor.

(3) Only one dimensionless parameter is scale-sensitive: the speed parameter $U=\eta u / 2 r E_{q}$ depends on the scale factor only for the term $r$. If we artificially modify another term $r$ (for example, by changing the viscosity from $\eta$ to $\eta S_{c}$ ), then this parameter, as all other parameters, remains independent of the scale factor.

Consequently, the resulting calculation of individual normal loads at asperities only depends on factor terms used to transform the dimensionless term into dimensional quantities. The proposed calculation for the friction coefficient is only based on an evaluation of normal loads (3). It is interesting to note that all normal loads on asperities are scaled by the square of the radius value and, consequently, by the square of the scale factor. The friction coefficient, as a ratio of loads, is not influenced by the scale parameter of the microgeometry. As a synthesis, if friction using the modified viscosity (viscosity $\eta$ is replaced by a new viscosity $\eta S_{c}$ ) is not modified by the scale factor, the result corresponding to the homothetic microgeometry and the initial viscosity is obtained with the $\eta u / p S_{c}$ value for the Stribeck parameter. Finally, if we adopt this modified Stribeck term, the friction coefficient is independent of the scale parameter, and it is logical to obtain the same curve for all homothetic geometries.

Because all expressions in the theoretical approach can be expressed with a dimensional parameters, existence of a master curve is not limited to friction coefficient evolution but also for fraction of force transmitted by each of the 5 families of asperity (Figure 9).

Figure 9 gives the fraction of normal load supported by each family of asperity as a function of the modified Stribeck parameter: $\eta \mathrm{u} / \mathrm{pS}_{\mathrm{c}}$. As evidence, because one asperity must be working with one of the 5 identified mechanisms, the summation of these fraction has to be always equal to $100 \%$. But repartition depends on the situation and in particular with the modified parameter $\eta \mathrm{u} / \mathrm{pS}_{\mathrm{c}}$. The considered situations in this study show that sharing of normal load is not uniform into all five mechanisms. Depending on lubrication condition expressed by the modified Stribeck parameter, one can observe 2 major mechanisms (Figure 9(a)):

(i) Elastoplastic mechanism for direct contact (ranging from 0 to $96 \%$ )

(ii) Hydrodynamic lubrication for lubricated contact (ranging from 0 to $100 \%$ )

As explained in [14], main reason for the existence of only 2 from the five possible mechanisms is the largescale between transitions from one mechanism to the next compared to the variability of situation due to the range of altitude for asperities. Other families mechanisms are identified on details of the global curve:

(i) Elastohydrodynamic lubrication (ranging from 0 to 18 $\%)$ 


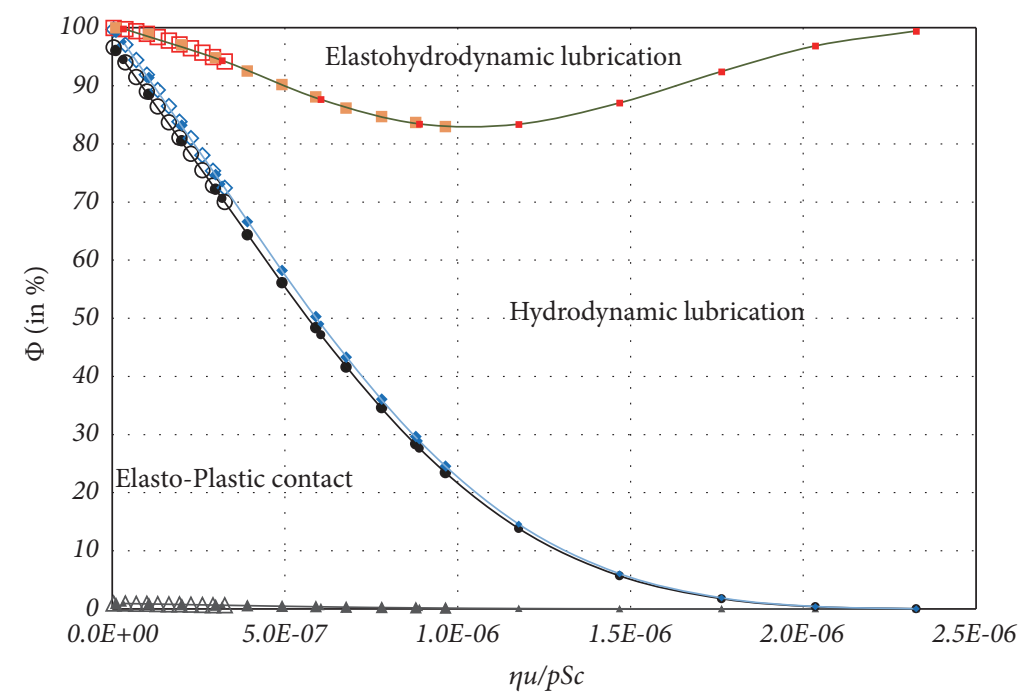

(a) Complete curve

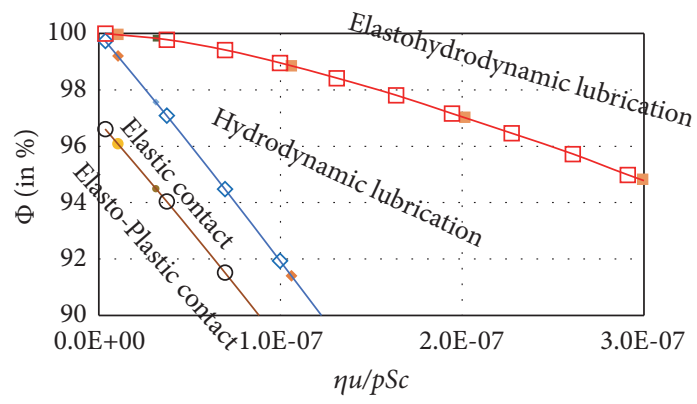

(b) Detail

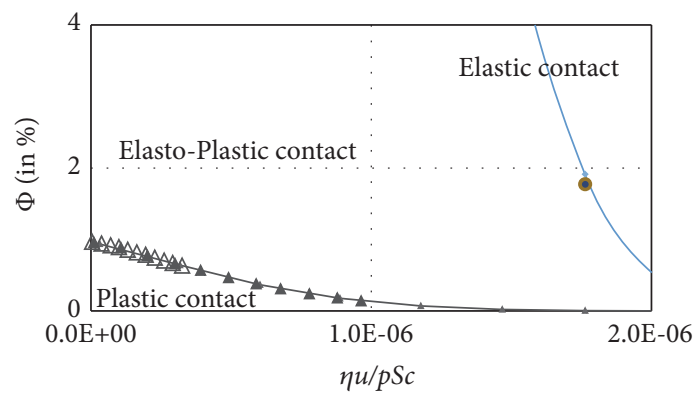

(c) Detail

FIGURE 9: Fraction of force transmitted by each of the 5 families of asperity ((a) global curve, (b) and (c) details).

(ii) Elastic mechanism (less than $3 \%$ in detail (b) of Figure 9)

(iii) Plastic deformation (less than $1 \%$ in detail (c) of Figure 9)

It is also important to note that Figure 9 regroups information obtained using 3 different microgeometries:

(i) Reference microgeometry represented by intermediate filled marks

(ii) Scaled microgeometry with a factor of $1 / 3$ represented by small filled marks

(iii) Scaled microgeometry with a factor of 3 represented by big marks

The use of the modified Stribeck parameter gives an excellent continuity for fraction of asperities working in the same condition. This confirms the existence, for all families of asperity, of a dimensionless formulation adapted to the modified parameter.

\section{Conclusions}

This article presented theoretical predictions for friction evolution under variation of contact parameters and microgeometry. The applied model was based on the decomposition of the contact in neighboring asperities. Depending on the contact parameters and the geometry of the considered asperities, the behavior was assigned to 5 possible regimes. The behavior of the contact was obtained by associating the elementary behavior determined for each asperity.

From a qualitative perspective, simulations using this model exhibit the classical decreasing Stribeck curve between the friction coefficient and the well-known parameter $\eta u / p$. We also observed a decrease in the friction coefficient with a decrease in the amplitude of the microgeometry. We have studied the behavior of homothetic microgeometries (deduced one from the other by the same amplification in all directions). All homothetic microgeometries give exactly the same behavior if the classical Stribeck parameter $\eta u / p$ is transformed into a new parameter $\eta u / p S_{c}$ that includes the scale factor $S_{c}$ of the microgeometry. From a quantitative perspective, this result is in very good agreement with the experimental results given in the literature.

From a practical perspective, this approach enables the prediction of the behavior of a family of new microgeometries from that obtained for a given single microgeometry. A necessary condition for the proposed extrapolation is to consider that the new geometry must be scaled from the initial one. A possible perspective of this work is to isolate the 
effects of an individual aspect of microgeometry (for example, only the vertical amplitude or periodicity).

\section{Nomenclature}

\begin{tabular}{|c|c|}
\hline$A_{0}:$ & Apparent area of contact \\
\hline$E_{i}:$ & Young's modulus of material "i” \\
\hline$E_{q}:$ & Equivalent Young's modulus \\
\hline$f_{i}^{4}(i=b, f):$ & $\begin{array}{l}\text { Coefficient of friction for boundary } \\
\text { lubrication (b) and for fluid } \\
\text { lubrication (f) }\end{array}$ \\
\hline$F_{\text {regime }}:$ & $\begin{array}{l}\text { Normal load of individual asperity } \\
\text { working in the corresponding regime } \\
\text { (subscript regime can be EL, EP, PL, } \\
\text { HL, and EHL) }\end{array}$ \\
\hline$G=\alpha E_{q}:$ & Material parameter \\
\hline$h:$ & Mean film thickness in the contact \\
\hline$h_{0}:$ & Local film thickness at an asperity \\
\hline$H_{0}=h_{0} / r:$ & Dimensionless film thickness \\
\hline$H_{0 E H L l i m}:$ & $\begin{array}{l}\text { Limiting film thickness in EHL } \\
\text { regime }\end{array}$ \\
\hline$H_{0 P V R \lim }:$ & $\begin{array}{l}\text { Limiting film thickness in PVR } \\
\text { regime }\end{array}$ \\
\hline$H p:$ & Constant used in EHL regime \\
\hline$m_{z}, s_{z}:$ & $\begin{array}{l}\text { Mean value of the summit altitude } \\
\text { and standard deviation }\end{array}$ \\
\hline$n_{0}:$ & $\begin{array}{l}\text { Total number of asperities on the } \\
\text { entire surface }\end{array}$ \\
\hline$N:$ & Normal load on the contact \\
\hline$N_{\text {regime }}:$ & $\begin{array}{l}\text { Normal load on the contact for the } \\
\text { considered regime (subscript regime } \\
\text { can be EL, EP, PL, HL, and EHL) }\end{array}$ \\
\hline$p:$ & Apparent pressure in the contact \\
\hline$r:$ & Radius of asperity \\
\hline$R p_{e}:$ & Yield stress of the softer material \\
\hline$R q:$ & $\begin{array}{l}\text { Standard deviation of the } \\
\text { microgeometry }\end{array}$ \\
\hline$R q_{\text {asper }}:$ & $\begin{array}{l}\text { Standard deviation of the microrelief } \\
\text { of asperities }\end{array}$ \\
\hline$S_{c}:$ & Scale factor of the microgeometry \\
\hline$T:$ & Friction force \\
\hline$u:$ & Sliding speed \\
\hline$U=\eta u / 2 r:$ & Dimensionless speed \\
\hline$W_{\text {mech }}=F_{\text {mech }} / E_{q} r^{2}:$ & Dimensionless normal load \\
\hline$z:$ & Altitude of summit \\
\hline$\alpha:$ & Piezoviscosity coefficient of lubricant \\
\hline$\beta:$ & Density of asperities \\
\hline$\delta:$ & Asperity indentation \\
\hline$\Delta=\delta / r$ & Dimensionless deformation \\
\hline$\Delta_{E L l i m}:$ & $\begin{array}{l}\text { Elastic/elastoplastic limit for } \\
\text { dimensionless deformation }\end{array}$ \\
\hline$\Delta_{E P \lim }:$ & $\begin{array}{l}\text { Elastoplastic/plastic limit for } \\
\text { dimensionless deformation }\end{array}$ \\
\hline$\eta:$ & Viscosity of lubricant \\
\hline$\phi:$ & $\begin{array}{l}\text { Ratio of load transmitted by } \\
\text { asperities working with local } \\
\text { boundary regime }\end{array}$ \\
\hline$v_{i}$ & Poisson coefficient of surface "i \\
\hline
\end{tabular}

\section{Data Availability}

The data used to support the findings of this study are available from the corresponding author upon request.

\section{Conflicts of Interest}

The authors, Francois Robbe-Valloire, Robert Progri, and Tony Da Silva Botelho, declare that there are no conflicts of interest regarding the publication of this paper.

\section{References}

[1] C. J. Hooke, “The effect of roughness in EHL contacts," Tribology and Interface Engineering Series, vol. 48, pp. 31-46, 2005.

[2] A. Beheshti and M. M. Khonsari, "An engineering approach for the prediction of wear in mixed lubricated contacts," Wear, vol. 308, no. 1-2, pp. 121-131, 2013.

[3] M. B. Dobrica, M. Fillon, and P. Maspeyrot, "Mixed elastohydrodynamic lubrication in a partial journal bearing - Comparison between deterministic and stochastic models," Journal of Tribology, vol. 128, no. 4, pp. 778-788, 2006.

[4] A. Albers and S. Reichert, "On the influence of surface roughness on the wear behavior in the running-in phase in mixedlubricated contacts with the finite element method," Wear, vol. 376-377, pp. 1185-1193, 2017.

[5] H. Zhang, M. Hua, G.-N. Dong, D.-Y. Zhang, and K.-S. Chin, "A mixed lubrication model for studying tribological behaviors of surface texturing," Tribology International, vol. 93, pp. 583-592, 2016.

[6] S. Fricke, C. Hager, S. Solovyev, M. Wangenheim, and J. Wallaschek, "Influence of surface form deviations on friction in mixed lubrication," Tribology International, vol. 118, pp. 491499, 2018.

[7] W. C. Emmens, "The influence of surface roughness on friction," in Proceedings of the 15th biennial Congress IDDRG at Dearborn, pp. 63-70, Michigan USA, May 1988.

[8] E. Felder and V. Samper, "The generation by friction and plastic deformation on the restraining characteristics of draw beads in sheet metal forming," in Proceedings of the 20th Leeds-Lyon Symposium on Tribology: Dissipative Processes in Tribology, pp. 361-372, Lyon, France, September 1993.

[9] I. Nogueira, A. M. Dias, R. Gras, and R. Progri, "An experimental model for mixed friction during running-in," Wear, vol. 253, no. 5-6, pp. 541-549, 2002.

[10] A. O. Lebeck, "Parallel sliding load support in the mixed lubrication - Part 2: Evaluation of the mechanisms," Journal of Tribology, vol. 109, pp. 196-205, 1987.

[11] S. Xiong and R. F. Salant, "A dynamic model of contacting mechanical seal for down-hole tools," Journal of Tribology, vol. 125, no. 2, pp. 391-402, 2003.

[12] J. Hol, V. T. Meinders, H. J. M. Geijselaers, and A. H. Van Den Boogaard, "Multi-scale friction modeling for sheet metal forming: The mixed lubrication regime," Tribology International, vol. 85, pp. 10-25, 2015.

[13] D. K. Karupannasamy, J. Hol, M. B. de Rooij, T. Meinders, and D. J. Schipper, "Modelling mixed lubrication for deep drawing processes," Wear, vol. 294-295, pp. 296-304, 2012.

[14] F. Robbe-Valloire and R. Progri, "Mechanisms developed at the asperity scale for mixed lubrication between parallel surfaces," 
Journal of Engineering Tribology, vol. 226, no. 12, pp. 1441-1453, 2012.

[15] F. Robbe-Valloire, B. Paffoni, and R. Progri, "Prediction of the amount of load transmitted by elastic, elastoplastic or plastic deformed asperities at a rough interface," Mechanics of Materials, vol. 33, no. 11, pp. 617-633, 2001.

[16] K. L. Johnson, J. A. Greenwood, and S. Y. Poon, "A simple theory of asperity contact in elastohydro-dynamic lubrication," Wear, vol. 19, no. 1, pp. 91-108, 1972.

[17] K. L. Johnson, "Normal contact of inelastic solids," in Contact Mechanics, Cambridge University Press, 1985.

[18] W. R. Chang, I. Etsion, and D. B. Bogy, "An elastic-plastic model for the contact of rough surfaces," Journal of Tribology, vol. 109, no. 2, pp. 257-263, 1987.

[19] L. Kogut and I. Etsion, "Elastic-plastic contact analysis of a sphere and a rigid flat," Journal of Applied Mechanics, vol. 69, no. 5, pp. 657-662, 2002.

[20] R. Jackson, I. Chusoipin, and I. Green, "A finite element study of the residual stress and deformation in hemispherical contacts," Journal of Tribology, vol. 127, no. 3, pp. 484-493, 2005.

[21] D. E. Brewe and B. J. Hamrock, "Analysis of starvation on hydrodynamic lubrication in non-conform contact," Journal of Lubrication Technology, vol. 104, no. 3, pp. 410-411, 1982.

[22] B. J. Hamrock and D. Dowson, "Isothermal elastohydrodynamic lubrication of points contacts," Journal of Lubrication Technology, vol. 99, pp. 264-274, 1977.

[23] J. A. Greenwood and J. B. Williamson, "Contact of nominally flat surfaces," Proceedings of the Royal Society A Mathematical, Physical and Engineering Sciences, vol. 295, no. 1442, pp. 300319, 1966.

[24] F. Robbe-Valloire, "Statistical analysis of asperities on a rough surface," Wear, vol. 249, no. 5-6, pp. 401-408, 2001. 


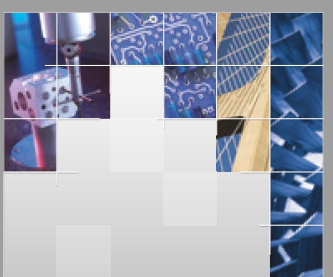

\section{Enfincering}
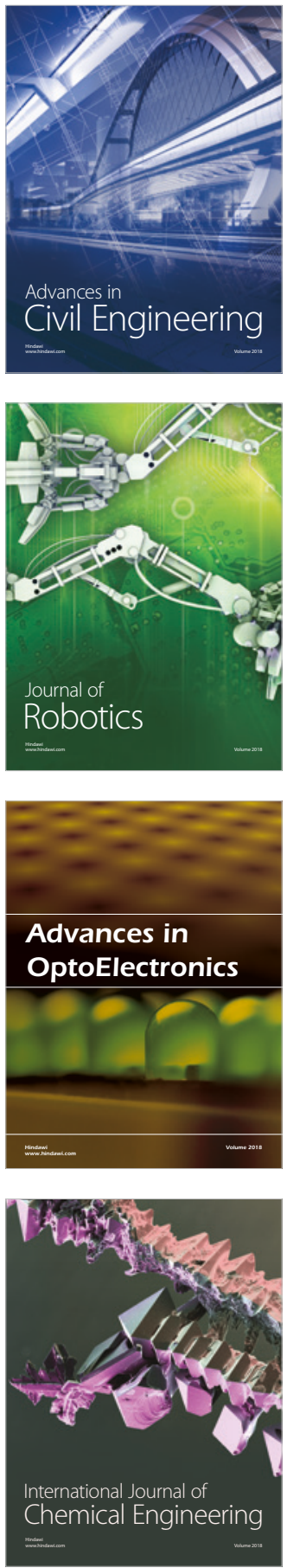

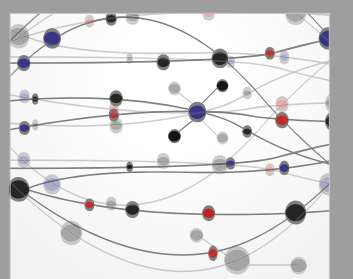

\section{Rotating \\ Machinery}

The Scientific World Journal

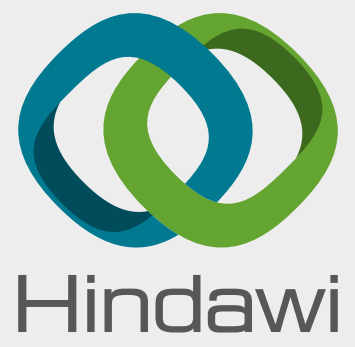

Submit your manuscripts at

www.hindawi.com
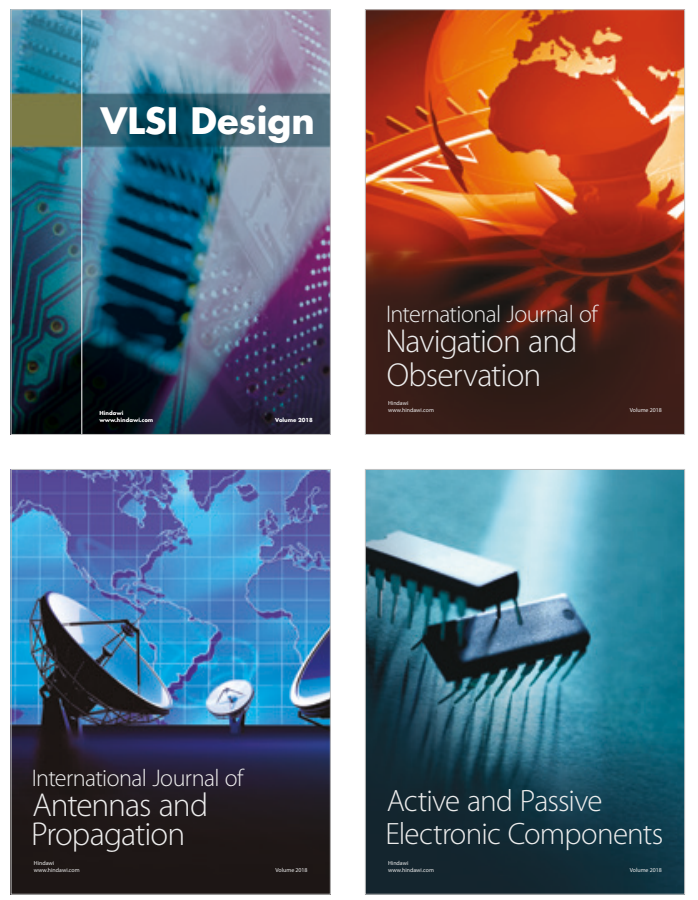
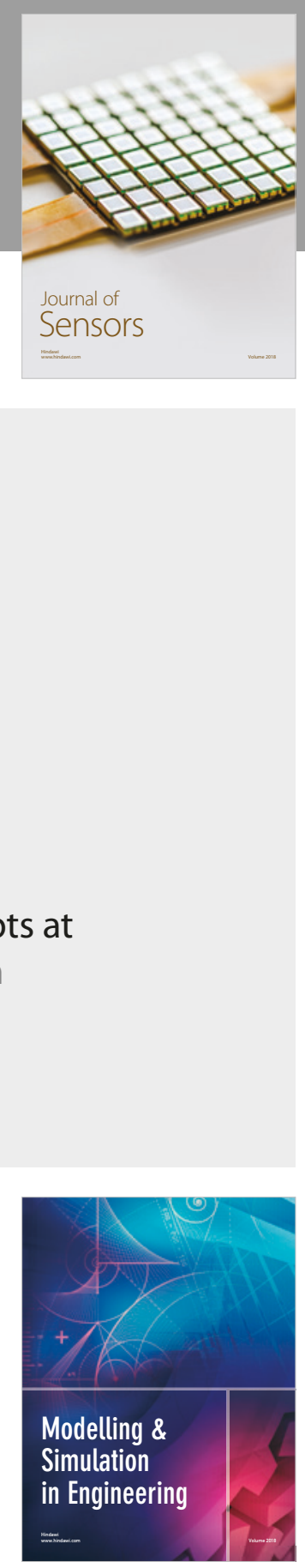

\section{Advances \\ Multimedia}
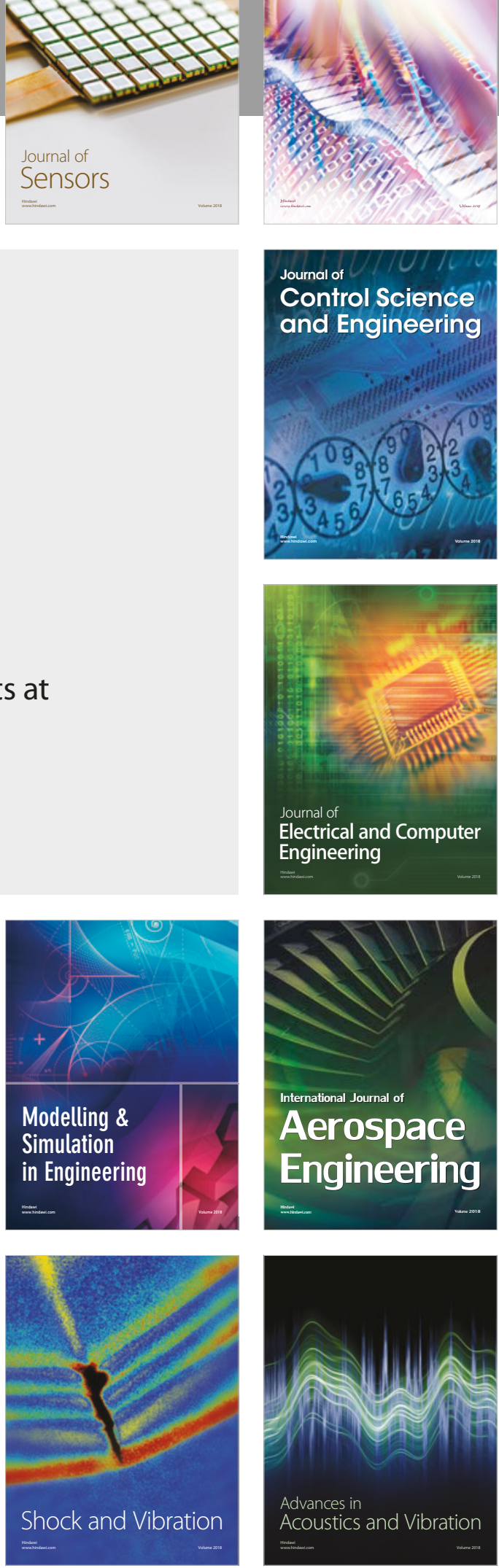